\title{
基于平面激光测量的移动机器人自定位方法
}

\author{
曾慧 ${ }^{2}$ 吴福朝 1 胡占义 ${ }^{1}$
}

\begin{abstract}
摘 要 提出了两种基于平面激光测量的移动机器人自定位方法. 第一种方法是改进的 Hough 密度谱的方法, 它的主要优点 是避免了现有方法 Hough 变换离散化过程中的信息损失问题, 提高了算法的精度和鲁棒性. 该方法在引进一种新的 Hough 密 度谱的基础上, 根据谱相关函数值和运动参数的密度得到机器人运动参数的候选值, 并应用 Hausdorff 相似性度量从候选值确 定运动参数的最终估计. 第二种方法是基于 Fourier-Mellin 变换的方法, 主要利用 Fourier 变换的位移理论和 Fourier-Mellin 不变量来估计运动参数. 为了避免图像离散化造成的信息损失, 在该方法中使用基于 Hausdorff 距离的最近点迭代 (ICP) 算 法来进一步精化平移向量. 实验结果表明, 这两种方法均可有效地提高机器人的定位精度, 具有一定的实际应用价值.
\end{abstract}

关键词 机器人自定位, Hough 密度谱, Fourier-Mellin 变换, Hausdorff 距离 中图分类号 TP24

\section{Robot Self-Localization Based on Planar Laser Measurement}

\author{
Zeng Hui ${ }^{1} \quad$ Wu Fu-Chao ${ }^{1} \quad \mathrm{Hu}$ Zhan-Yi ${ }^{1}$
}

\begin{abstract}
This paper presents two novel robot self-localization methods based on planar laser measurement. The first one is an improved Hough density spectrum based method, into which a novel Hough density spectrum is introduced, and by which the location accuracy and robustness can be both enhanced. The key advantageous aspect of our new spectrum is that its implementation does not involve any discretization error in the Hough space, which is the major source of location inaccuracy in the conventional method. The second one is an Fourier-Mellin transform based method. This method first converts the two measurement point sets into two binary images, then uses the Fourier-Mellin based image matching technique, a popular technique in image matching field, to determine the rotation parameter and finally invokes a standard ICP technique with the Hausdorff distance as its cost to estimate the two translation parameters. Experimental results show that both methods can perform robustly and accurately.
\end{abstract}

Key words Robot self-localization, Hough density spectrum, Fourier-Mellin transform, Hausdorff distance

\section{1 引言}

目前, 对于室内移动机器人的研究主要集中在 导航、地图创建和路径规划等几个方面, 其中最基本 的问题是机器人的定位问题. 定位是机器人利用先 验环境的地图信息和传感器 (里程计、视觉、声纳、 激光等) 测量数据等信息确定其在工作环境中所处 位置的过程, 它是使机器人具有自主能力的基本功 能之一, 因此具有重要的研究意义.

本文主要研究基于平面激光测量的机器人定位 问题. 该问题可以描述为通过匹配两组二维激光测 量点 (即参考数据和当前数据) 来计算它们之间的二 维刚体变换 $(R, T)$ 以决定机器人当前位姿的过程.

\footnotetext{
收稿日期 2005-9-6 收修改稿日期 2006-5-11

Received September 6, 2005; in revised form May 11, 2006 国家自然科学基金 (60303021), 国家高技术研究发展 (863) 计划 (2005AA118020) 资助

Supported by National Natural Science Foundation of P. R. China (60303021), the National High-Tech Research and Development Plan of P. R. China (2005AA118020)

1. 中国科学院自动化研究所模式识别国家重点实验室 北京 100080

1. National Laboratory of Pattern Recognition, Institute of Automation, Chinese Academy of Sciences, Beijing 100080 DOI: $10.1360 /$ aas-007-0138
}

在理想的结构化环境中, 基于特征的方法可以快速 准确地解决这一问题 ${ }^{[1,2]}$. 然而, 在非结构化环境 中, 由于很难提取环境的几何特征而导致该方法不 可用. 已有的解决方法是通过寻找待匹配测量点的 最近点并最小化距离误差来估计机器人的位姿, 即 经典的最近点迭代 (Iterative closest point, ICP) 算 法, 它通常需要里程计等传感器给出一个初始估计 才能使算法收玫. 在噪声相对较低且遮挡不严重的 情况下, 最近点迭代算法可得到较好的结果; 但是在 噪声比较高或者存在较严重遮挡的情况下, 使用最 近点迭代算法进行激光测量点匹配存在易陷入局部 最小点的问题 [3,4]. Andrea Censi 等在文献 [5] 中 通过 Hough 变换将这个时域问题转化为频域问题, 然后使用谱分析方法来解决. 实验结果表明谱分析 方法不需要初始估计, 不存在特征提取和确定对应 关系的问题, 既可用于结构化环境又可用于非结构 化环境且对噪声不敏感, 具有较高的定位精度. 这种 方法的不足之处是其中 Hough 变换的离散化会造 成一定的信息损失.

本文探讨了两种基于平面激光测量的机器人自 定位方法. 其中一种方法是改进的 Hough 密度谱 
的方法, 通过定义一种新的 Hough 密度谱和估计 运动参数的概率密度来求解 $(R, T)$, 既保持了文献 [5] 中方法的优点又有效地避免了 Hough 变换离散 化的信息损失, 提高了算法的精度和鲁棒性. 另一 种方法是基于 Fourier-Mellin 变换的定位方法. 该 方法首先利用 Fourier 变换的位移理论和 FourierMellin 不变量来估计运动参数 $(R, T)$, 然后使用基 于 Hausdorff 距离的 ICP 算法来进一步精化平移向 量 $T$.

本文组织结构如下: 第 2 节是对基于平面激 光测量的机器人自定位方法的问题描述; 第 3 节 介绍改进的 Hough 密度谱方法; 第 4 节讨论基于 Fourier-Mellin 变换的定位方法; 第 5 节是实验结 果; 第 6 节是本文的结论.

\section{2 问题描述}

基于平面激光测量的机器人自定位方法与基于 视觉、声纳等传感器的定位方法相比具有精度高、 计算量小等优点, 得到了广泛的应用 $[2,3]$. 激光测距 仪发射出的激光束经测量物体反射后又被测距仪接 收, 测距仪同时记录激光往返的时间. 光速和往返 时间乘积的一半就是测距仪和被测量物体之间的距 离. 随着激光测距仪发射出的激光在其扫描平面上 的旋转, 测距仪得到位于被测环境与扫描平面交线 上的一组距离测量点. 激光测量点可通过一个以测 距仪所在位置为原点、首次激光发射的方向为极轴 的极坐标系来描述, 即每一个激光测量点都由激光 发射的方向和测量距离来表示.

在本文中, 我们假设安装在机器人上的激光测 距仪的扫描平面是水平的. 若机器人从一个位置水 平移动到另外一个位置, 则激光测距仪可得到位于 同一个环境平面上的两组距离测量点, 因此利用激 光测距仪进行机器人定位的问题可转化为同一平面 两组点的匹配问题. 这个匹配问题可以描述为: 给定 机器人运动前后的两组激光测量点 $P_{1}, P_{2}, \cdots, P_{n}$ 和 $P_{1}^{\prime}, P_{2}^{\prime}, \cdots, P_{n}^{\prime}$, 求运动参数 $(R, T)$ 以使这两组 激光测量点尽可能地对准. 这里, 两组待匹配点的对 应关系未知, 即点 $P_{i}$ 与 $P_{i}^{\prime}$ 并不一定为对应点. 因 为机器人运动会改变激光测距仪的测量环境, 所以 这两组待匹配激光测量点所对应的测量环境并不完 全相同, 只有部分是重合的. 也就是说, 仅仅有一部 分待匹配点能够在另外一组待匹配点中找到对应点, 并且这些确定存在对应的待匹配点之间的对应关系 未知. 由于激光测距仪有随机测量误差且环境中可 能会存在遮挡, 因此我们不可能将这两组激光测量 点精确地对准. 最后, 需要指出的是, 从理论上来说, 第 3 节将要介绍的基于 Hough 密度谱的方法要求两
组测量点集存在严格的对应关系, 但不需要知道两 组点集内点与点之间的对应关系. 在实际具体应用 中, 事实上两组测量点集之间不可能存在严格的对 应关系, 有时甚至两组点集的个数也不可能完全相 同，但有一个对应子集确实存在对应关系. 在这种情 况下, 基于 Hough 密度谱的方法本质上是利用该对 应子集来进行定位, 测量点集中其它不存在对应的 点均可以看作 “噪声点”。由于经过 Hough 变换后 这些噪声点在参数空间的映射不可能聚类, 所以这 种噪声一般来说对最终定位的精度不会产生太大的 影响. 当然, 这取决于 “噪声点” 的比例、分布以及 所定义的密度谱.

\section{3 改进的 Hough 密度谱方法}

\subsection{Hough 变换的密度谱}

假设点 $P$ 经过刚体变换 $(R, T)$ 被映射到点 $P^{\prime}$, 即 $P^{\prime}=R P+T$. 若点 $P$ 和 $P^{\prime}$ 所对应的 Hough 变 换的值分别记为 $H T(\theta, \rho)$ 和 $H T^{\prime}(\theta, \rho)$, 旋转角记 为 $\phi$, 则根据 Hough 变换的定义有下式 [6]

$$
\left.H T^{\prime}(\theta, \rho)=H T(\theta+\phi, \rho+(\cos \theta, \sin \theta) T)\right]
$$

由式 (1) 可以得到我们将要用到的 Hough 变换的重 要性质, 即点 $P$ 所对应的 Hough 变换在 $\theta$ 处的 $\rho$ 值 与点 $P^{\prime}$ 所对应的 Hough 变换在 $(\theta+\phi)$ 处的 $\rho$ 值 相差相同的平移 $(\cos \theta, \sin \theta) T$. 也就是说, 空间点 在变换前所对应的 Hough 变换在 $\theta$ 处 $\rho$ 值的相对分 布与它们变换后所对应的 Hough 变换在 $(\theta+\phi)$ 处 的 $\rho$ 值的相对分布相同.

假设函数 $g$ 具有平移不变性, 即若 $f^{\prime}(\pi)=$ $f(\pi+a)$, 则 $g[f]=g\left[f^{\prime}\right]$. 那么我们定义点集 $P_{1}, P_{2}, \cdots, P_{n}$ 所对应的 Hough 密度谱为

$$
H S(\theta):=g[H T(\theta, \cdot)]
$$

其中, $H T(\theta, \cdot)$ 表示由点集 $P_{1}, P_{2}, \cdots, P_{n}$ 所对应 的 Hough 变换在 $\theta$ 处的 $\rho$ 值 $\rho_{1}, \rho_{2}, \cdots, \rho_{n}$ 的某种 函数式组成的向量. 因为正弦曲线的周期为 $2 \pi$, 所 以这里 $\theta$ 只在 $[0,2 \pi]$ 之间取值. 若两组待匹配点 $P_{1}, P_{2}, \cdots, P_{n}$ 和 $P_{1}^{\prime}, P_{2}^{\prime}, \cdots, P_{n}^{\prime}$ 所对应的 Hough 变换满足关系式 $H T(\theta, \cdot)=H T^{\prime}(\theta+\phi, \cdot)$, 则由 Hough 密度谱的定义可知它们所对应的 Hough 密 度谱 $H S(\theta) 、 H S^{\prime}(\theta+\phi)$ 相等, 即 $H S(\theta)=H S^{\prime}(\theta+$ $\phi)$, 该性质称为 Hough 密度谱的平移不变性.

\section{2 基于 Hough 密度谱的定位方法}

文献 [5] 给出了一种基于 Hough 密度谱的机器 人定位方法, 其中 Hough 变换采用的是离散 Hough 变换. 其具体实现步骤如下: 首先, 选择合适的 $\rho 、 \theta$ 的最大值和最小值, 并对 Hough 空间进行离散化处 
理. 然后, 建立一个二维累加器 $A(\rho, \theta)$, 且使它的 每一个元素的初始值均为 0 . 累加器矩阵的大小依 赖于 Hough 空间离散化的程度. 最后, 对输入空间 的每一点 $P_{i}$ 计算曲线 $\rho=(\cos \theta, \sin \theta) P_{i}$, 将累加 器 $A(\rho, \theta)$ 对应的元素加 1 .

假设相差一个刚体变换 $(R, T)$ 的点集 $P_{1}, P_{2}, \cdots, P_{n}$ 和 $P_{1}^{\prime}, P_{2}^{\prime}, \cdots, P_{n}^{\prime}$ 对应的累加器矩阵 分别为 $A(\rho, \theta)$ 和 $A^{\prime}(\rho, \theta)$, 矩阵 $A$ 的第 $\theta$ 列的非零 元素表示为 $e=\left[\boldsymbol{e}_{1}, \boldsymbol{e}_{2}, \cdots, \boldsymbol{e}_{m}\right]^{\mathrm{T}}$, 矩阵 $A^{\prime}$ 第 $\theta+\phi$ 列的非零元素表示为 $e^{\prime}=\left[\boldsymbol{e}_{1}^{\prime}, \boldsymbol{e}_{2}^{\prime}, \cdots, \boldsymbol{e}_{m}^{\prime}\right]^{\mathrm{T}}$. 则由 3.1 节 Hough 变换的定义可知, $e=e^{\prime}$. 因此, 文献 $[5]$ 选取的 $H T(\theta,:)=e$ 满足 $H T(\theta, \cdot):=H T^{\prime}(\theta+$ $\phi, \cdot)$ 的要求. 文献 $[5]$ 选取函数 $g[f]=\sum f_{i}^{2}$ 来定义 具有平移不变性的 Hough 密度谱, 即

$$
H S(\theta):=\sum_{i=1}^{m} e_{i}^{2}
$$

这里定义的 Hough 密度谱的周期为 $\pi$. 由于 Hough 密度谱满足平移不变性, 故通过对它们作相关即可 估计出旋转角 $\phi$. 定义两组待匹配激光测量点所对 应的 Hough 密度谱 $H S(\theta)$ 和 $H S^{\prime}(\theta)$ 之间的相关 函数为

$$
\operatorname{Corr}(\phi)=\sum_{\theta \in \Theta} H S(\theta) \cdot H S^{\prime}(\theta-\phi)
$$

通过求该相关函数的局部最大值可得到一组旋转角 $\phi$ 的候选值 $\left\{\phi_{1}, \phi_{2}, \cdots, \phi_{k}\right\}$. 由于 Hough 密度谱的 周期性和各种误差, 出现多个候选值是可能的. 对 每一个旋转角候选值 $\phi_{i}$, 将第一组激光测量点进行 旋转得到点集 $P_{1}^{\phi}, P_{2}^{\phi}, \cdots, P_{n}^{\phi}$, 则它与第二组激光 测量点仅相差一个平移, 即它们所对应的 Hough 变 换满足下列关系

$$
H T(\theta, \rho)=H T^{\phi}(\theta, \rho+(\cos \theta, \sin \theta) T)
$$

那么我们就可以把求解平移向量 $T$ 的问题转化为一 个最小二乘问题. 即对每一个 $\theta_{i}$ 均有

$$
\left(\cos \theta_{i}, \sin \theta_{i}\right) T=d\left(\theta_{i}\right) \quad i=1, \cdots, n
$$

显然, 只要满足 $n \geq 2$ 就可得到 $T$ 的一组解. 由于机器人运动前后的激光测量点的对应关系未 知, 故不能直接得到 $d\left(\theta_{i}\right)$ 的值. 通过对 $H T\left(\theta_{i}, \cdot\right)$ 和 $H T^{\phi}\left(\theta_{i}, \cdot\right)$ 作相关可求解 $d\left(\theta_{i}\right)$, 其中 $\theta_{i}$ 取第二组激 光测量点所对应的 Hough 密度谱的局部最大值.

通过以上对文献 [5] 所述方法的分析可以看出: 由于累加器是对 Hough 变换进行离散化处理而得 到的, 不可避免地会有一些信息损失; 另一方面, 只 有当平移量 $(\cos \theta, \sin \theta) T$ 为 $\rho$ 的离散化单位量的 整数倍时, Hough 密度谱才能严格满足平移不变性.
因此文献 [5] 的方法虽然可以在一定程度上提高算 法的精度和鲁棒性, 但仍有一定的不足之处.

\section{3 改进的 Hough 密度谱方法}

\subsection{1 改进的 Hough 密度谱}

为了克服 Hough 变换离散化造成的信息损失, 进一步提高算法的精度, 我们提出一种不需要对 Hough 变换的 $\rho$ 值进行离散化的 Hough 密度谱. 首先, 我们计算向量 $h=\left[\boldsymbol{\rho}_{1}-\overline{\boldsymbol{\rho}}, \boldsymbol{\rho}_{2}-\overline{\boldsymbol{\rho}}, \cdots, \boldsymbol{\rho}_{n}-\right.$ $\overline{\boldsymbol{\rho}}]$, 其中 $\bar{\rho}=\left(\sum \rho_{i}\right) / n$ 为 $\rho$ 的中心点; 然后选取 $H T(\theta, \cdot):=h$. 由于两组待匹配激光测量点所对应 的 Hough 变换在任意 $\theta$ 和 $\theta+\phi$ 处对应的 $\rho$ 值相 差相同的平移 $(\cos \theta, \sin \theta) T$, 也就是说它们相对于 各自中心点的分布相同, 因而可以推出 $H T(\theta, \cdot)=$ $H T^{\prime}(\theta+\phi, \cdot)$. 我们仍然用函数 $g[f]=\sum f_{i}^{2}$ 来定义 新的 Hough 密度谱, 即

$$
H S(\theta):=\sum_{i=1}^{n}\left(\rho_{i}-\bar{\rho}\right)^{2}
$$

由上式可以看出, $H S(\theta)$ 仅与 $\rho_{i}$ 相对于其中心 点 $\bar{\rho}$ 的分布有关, 而与 $\rho_{i}$ 的具体值的大小无关. 因 此两组待匹配激光测量点所对应的 Hough 密度谱 $H S(\theta)$ 和 $H S^{\prime}(\theta)$ 满足平移不变性. 与文献 [5] 的方 法相比, 本文定义的新的 Hough 密度谱不但保持了 平移不变性, 而且不需要对 Hough 变换的 $\rho$ 值进行 离散化, 不需要计算累加器矩阵, 大大减小了计算 量, 提高了算法的精度.

\subsection{2 旋转角 $\phi$ 和平移向量 $T$ 的估计}

对于旋转角 $\phi$ 的估计, 文献 [5] 是通过求相关 函数的局部最大值来得到一组旋转角 $\phi$ 的候选值 $\left\{\phi_{1}, \phi_{2}, \cdots, \phi_{k}\right\}$. 由于相关函数在局部最大值附近 的点比较密集, 故本文通过估计相关函数值在稠 密区间的密度 (即旋转角 $\phi$ 在稠密区间的密度) 来 提高 $\phi$ 的精度和算法的鲁棒性. 方法如下：首先， 检测相关函数点比较密集的区间 $I_{1}, I_{2}, \cdots, I_{k}$, 并 记稠密区间 $I_{i}(i=1,2, \cdots, k)$ 中相关函数的点为 $\left(\boldsymbol{\alpha}_{1}^{i}, \boldsymbol{r}_{1}^{i}\right),\left(\boldsymbol{\alpha}_{2}^{i}, \boldsymbol{r}_{2}^{i}\right), \cdots,\left(\boldsymbol{\alpha}_{m}^{i}, \boldsymbol{r}_{m}^{i}\right)$, 其中 $\left(\boldsymbol{\alpha}_{j}^{i}, \boldsymbol{r}_{j}^{i}\right)$ 表示 在区间 $I_{i}$ 中 $\alpha_{j}^{i}$ 处相关值为 $r_{j}^{i}$ 的点; 然后, 定义点 $\left(\boldsymbol{\alpha}_{t}^{i}, \boldsymbol{r}_{t}^{i}\right)(t=1,2, \cdots, m)$ 的密度为

$$
u_{t}^{i}=\left|r_{t}^{i}\right| / \sum_{j=1}^{m}\left|t_{j}^{i}\right|
$$

从公式 (8) 可以看出, $\left|r_{t}^{i}\right|$ 的值越大, 该点越接近局 部最大点, 它对应的密度也就越大. 最后, 利用下面 的公式得到旋转角 $\phi$ 的候选值 $\phi_{i}$

$$
\phi_{i}=\sum_{t=1}^{m} \alpha_{t}^{i} \times u_{t}^{i}
$$


这样, 对 $k$ 个稠密区间, 我们可得到旋转角 $\phi$ 的 $k$ 个候选值 $\left\{\phi_{1}, \phi_{2}, \cdots, \phi_{k}\right\}$.

关于平移向量 $T$ 的估计, 文献 [5] 通过选择 Hough 密度谱的局部最大值对应的 $\theta_{i}$ 来求解. 为了 进一步减少噪声对算法的影响, 我们采取与上面估 计旋转角 $\phi$ 类似的方法: 对每一个旋转角候选值 $\phi_{i}$, 首先检测出第二组激光数据所对应的 Hough 密度 谱点比较密集的区间 $I_{1}, I_{2}, \cdots, I_{k}$, 从任意两相邻 区间中分别取一个点可得到一组方向 $\left(\theta_{i}, \theta_{j}\right)$, 重复 取多次可得到多组方向. 由于 Hough 密度谱的周期 为 $\pi$, 为了减少计算量, 我们只取前两个相邻区间即 可. 对每一组方向利用公式 (6) 均可求出一组平移向 量 $T$, 故我们可得到 $T$ 的多组解. 估计每组解对应 的密度, 利用与公式 (9) 类似的形式可最终求出平 移向量 $T$ 的一组候选值 $T_{i}$.

对求得的多组 $(\phi, T)$ 的候选值, 我们需要从中 选出一组解作为运动参数的最终估计. 由于 Hausdorff 距离是比欧氏距离更鲁棒的一种相似性度量, 因此我们选择 Hausdorff 距离来衡量两组待匹配点 的对准程度. 对每组候选值 $\left(\phi_{i}, T_{i}\right)$, 将第一组激光 测量点 $P_{1}, P_{2}, \cdots, P_{n}$ 利用公式 $P^{\prime \prime}=R P+T$ 进行 变换得到点集 $P_{1}^{\prime \prime}, P_{2}^{\prime \prime}, \cdots, P_{n}^{\prime \prime}$. 在不考虑外点的前 提下, 计算 $P_{1}^{\prime}, P_{2}^{\prime}, \cdots, P_{n}^{\prime}$ 与 $P_{1}^{\prime \prime}, P_{2}^{\prime \prime}, \cdots, P_{n}^{\prime \prime}$ 之间 的 Hausdorff 距离. 具有最小 Hausdorff 距离的那组 候选值即为待求运动参数的最终估计 $\left(\phi^{*}, T^{*}\right)$.

\subsection{3 改进的 Hough 密度谱算法}

1) 计算两组待匹配激光测量点 $P_{1}, P_{2}, \cdots, P_{n}$ 和 $P_{1}^{\prime}, P_{2}^{\prime}, \cdots, P_{n}^{\prime}$ 的 Hough 变换及 Hough 密度谱.

2) 估计相关函数值在稠密区间的密度, 求出 $\phi$ 的候选值 $\left\{\phi_{1}, \phi_{2}, \cdots, \phi_{k}\right\}$.

3) 对每一个 $\phi_{i}$,

a) 将 $P_{1}, P_{2}, \cdots, P_{n}$ 旋转 $\phi_{i}$ 得到点集 $P_{1}^{\phi}, P_{2}^{\phi}, \cdots, P_{n}^{\phi}$.

b) 选出一组方向 $\left(\theta_{i}, \theta_{j}\right)$, 利用公式 (6) 求出 $T$ 的一组解

c) 重复 b), 直至取遍所有组方向, 得到 $T$ 的 多组解.

d) 估计每组解对应的密度, 求出一组候选值 $T_{i}$.

4) 重复 3), 可得到 $T$ 的候选值 $T_{1}, T_{2}, \cdots, T_{k}$.

5) 利用 Hausdorff 距离确定运动参数的最终估 计 $\left(\phi^{*}, T^{*}\right)$.

\section{4 基于 Fourier-Mellin 变换的定位方 法}

\section{1 基本原理}

基于 Fourier-Mellin 变换的匹配方法具有对噪 声不敏感、准确性高等优点, 是一种较为有效的匹 配方法. 文献中已有人把 Fourier-Mellin 变换应用 到图像匹配、曲线匹配等领域中 $[7 \sim 9]$, 我们把它 的应用范围拓展到机器人自定位中. 它的基本原 理是利用 Fourier 变换的位移理论和相似变换下的 Fourier-Mellin 不变量来进行匹配.

假设图像 $f_{2}(u, v)$ 为图像 $f_{1}(u, v)$ 在 $u$ 和 $v$ 方 向上分别平移 $u_{0}$ 和 $v_{0}$ 后的图像, 即

$$
f_{2}(u, v)=f_{1}\left(u-u_{0}, v-v_{0}\right)
$$

那么, 它们的 Fourier 变换满足下列关系

$$
F_{2}(\xi, \eta)=e^{-j \cdot \varphi(\xi, \eta)} \times F_{1}(\xi, \eta)
$$

则图像 $f_{1}(u, v)$ 和 $f_{2}(u, v)$ 的互功率谱为

$$
\frac{F_{1}(\xi, \eta) F_{2}^{*}(\xi, \eta)}{\left|F_{1}(\xi, \eta) F_{2}(\xi, \eta)\right|}=e^{\varphi(\xi, \eta)}
$$

其中, $F^{*}$ 为 $F$ 的共轭. 对 (12) 式进行 Fourier 反变 换, 将在 $\left(u_{0}, v_{0}\right)$ 处得到一个脉冲函数, 根据该脉冲 的位置即可确定两幅图像的平移量 $u_{0}$ 和 $v_{0}{ }^{[10]}$. 这 就是 Fourier 变换的位移理论.

若两幅图像 $f_{1}(u, v)$ 和 $f_{2}(u, v)$ 满足下列平移 和旋转变换关系

$$
\begin{array}{r}
f_{2}(u, v)=f_{1}\left(u \cos \phi+v \sin \phi+t_{u},\right. \\
\left.-u \sin \phi+v \cos \phi+t_{v}\right)
\end{array}
$$

根据 Fourier 变换的位移特性和旋转特性, 它们的 Fourier 变换可由下式表示

$$
\begin{array}{r}
F_{2}(\xi, \eta)=e^{j \cdot \varphi(\xi, \eta)} \times F_{1}(\xi \cos \phi+\eta \sin \phi, \\
-\xi \sin \phi+\eta \cos \phi)
\end{array}
$$

令 $M_{1} 、 M_{2}$ 分别为 $F_{1}$ 和 $F_{2}$ 的幅值, 则有下式成立

$$
\begin{array}{r}
M_{2}(\xi, \eta)=M_{1}(\xi \cos \phi+\eta \sin \phi, \\
-\xi \sin \phi+\eta \cos \phi)
\end{array}
$$

由式 (15) 可以看出, 两幅图像 Fourier 变换的 幅值只与两幅图像之间的旋转有关, 而与两幅图 像的平移无关, 该性质称为相似变换下的 FourierMellin 不变量 ${ }^{[10]}$.

\section{2 定位方法描述}

假设机器人在经过刚体运动 $(R, T)$ 前后的激 光测量点分别为 $P_{1}, P_{2}, \cdots, P_{n}$ 和 $P_{1}^{\prime}, P_{2}^{\prime}, \cdots, P_{n}^{\prime}$. 由于激光测量点都在同一个平面上, 因此我们可 把这两组激光测量点变成两幅二值图像 $f_{1}(u, v)$ 和 $f_{2}(u, v)$ (如可令测量点为 1 , 其它点均为 0 ), 且使这 两幅图像的尺度和大小均相等. 这样, 两组激光测 量点的匹配问题就转化为两幅二值图像的匹配问题. 
表 1 四种定位方法实验结果的比较

Table 1 Comparison of experimental results using four localization methods

\begin{tabular}{ccccc}
\hline & 平移误差 $\mathrm{x}(\mathrm{m})$ & 平移误差 $\mathrm{y}(\mathrm{m})$ & 旋转角误差 $(\mathrm{o})$ & Hausdorff 距离 $(\mathrm{m})$ \\
\hline HSM & 0.0171 & 0.0185 & 0.3642 & 0.0657 \\
IHSM & 0.0047 & 0.0023 & 0.1031 & 0.0309 \\
FM & 0.0163 & 0.0189 & 0.0698 & 0.0497 \\
FMH & 0.0057 & 0.0021 & 0.0698 & 0.0284 \\
HSM & 0.0390 & 0.0230 & 0.6843 & 0.0846 \\
IHSM & 0.0226 & 0.0198 & 0.2485 & 0.0539 \\
FM & 0.0420 & 0.0215 & 0.1325 & 0.0971 \\
FMH & 0.0216 & 0.0167 & 0.1325 & 0.0567 \\
& & & 2.3520 & 0.1089 \\
HSM & 0.0506 & 0.0470 & 1.6751 & 0.0764 \\
IHSM & 0.0369 & 0.0319 & 0.8763 & 0.1215 \\
FM & 0.0713 & 0.0364 & 0.8763 & 0.0734 \\
FMH & 0.0334 & 0.0328 & & \\
\hline
\end{tabular}

现用极坐标来表示两幅图像的频域幅值, 那么 (15) 式可以表示为下列形式

$$
M_{1}(\rho, \theta)=M_{2}(\rho, \theta-\phi)
$$

根据 Fourier 变换的位移理论, 我们首先对 $M_{1}(\rho, \theta)$ 和 $M_{2}(\rho, \theta-\phi)$ 作 Fourier 变换, 然后再计算它们对 应互功率谱的 Fourier 反变换, 最后根据得到的脉冲 函数的脉冲位置可求得旋转角 $\phi^{[10]}$.

对图像 $f_{1}(u, v)$ 绕其图像中心点旋转 $\phi$ 得到图 像 $f_{\phi}(u, v)$, 则 $f_{\phi}(u, v)$ 与图像 $f_{2}(u, v)$ 仅相差一个 平移, 即 $f_{2}(u, v)=f_{\phi}\left(u+t_{1}, v+t_{2}\right)$. 我们再次利 用 Fourier 变换的位移理论可求出图像在图像坐标 系中关于它们图像中心点的平移 $t_{1}$ 和 $t_{2}$, 利用坐标 系间的变换关系最终可求出机器人在世界坐标系下 的平移向量 $T$.

由于图像的离散化会造成一定的信息损失, 这 就不可避免地会影响到上述方法的精度, 尤其是对 平移向量 $T$ 的影响较大. 鉴于此我们又提出第二种 平移向量 $T$ 的估计方法, 即使用基于 Hausdorff 距 离的 ICP 算法. 它直接对激光数据进行处理, 能够 有效地提高算法的精度.

最近点迭代 (ICP) 算法是匹配激光测量点的经 典算法, 它通常需要有一个好的初始估计才能使算 法收玫. 当噪声比较高或者存在较多遮挡的情况下, 直接使用该算法往往得不到很好的结果. 如果我们 对只相差一个平移的两组数据进行匹配, 用 ICP 算 法一般可取得较好的结果. 由于 Hausdorff 距离能够 更好地度量两组待匹配点集的相似性, 故我们用基 于 Hausdorff 距离的 ICP 算法来估计平移向量 $T$.

在求出旋转角 $\phi$ 后, 我们将第一组激光测量点 $P_{1}, P_{2}, \cdots, P_{n}$ 进行旋转得到点集 $P_{1}^{\phi}, P_{2}^{\phi}, \cdots, P_{n}^{\phi}$, 则其与第二组激光测量点 $P_{1}^{\prime}, P_{2}^{\prime}, \cdots, P_{n}^{\prime}$ 仅相差一
个平移. 点集 $P^{\phi}$ 与 $P^{\prime}$ 之间的 Hausdorff 距离定义 为

$$
H\left(P^{\phi}, P^{\prime}\right)=\max \left\{h\left(P^{\phi}, P^{\prime}\right), h\left(P^{\prime}, P^{\phi}\right)\right\}
$$

其中, $h\left(P^{\phi}, P^{\prime}\right)=\max _{a \in P^{\phi}} \min _{b \in P^{\prime}}\|a-b\|,\|\cdot\|$ 表示两个点之间的欧氏距离 ${ }^{[11]}$.

我们使用 Hausdorff 距离定义待优化的目标函 数如下

$$
\min Z=H\left(P^{\phi}+T, P^{\prime}\right)+H\left(P^{\phi}, P^{\prime}-T\right)
$$

给定平移向量 $T$ 的初始值, 我们可通过搜索两个待 匹配点集之间的最近点对来重新估计 $T$, 反复迭代 直至算法收敛。

\section{5 实验和比较}

由于不能够精确测量出机器人的位置, 我们对 本文所述两种方法分别进行了模拟实验和实物实验, 并与文献 [5] 中的方法进行了比较, 实验结果表明本 文所述的两种方法均可有效地提高机器人的定位精 度.

\section{1 模拟实验}

首先从 Radish 机器人数据库 [12] 中下载 Stage 软件, 然后分别利用 Richard Vaughan 提供的医院 环境地图和手画洞穴环境地图 [13] 以及从 Texas 大 学网站上下载的 Cicurina 洞穴的环境地图 [14] 获取 三组模拟数据来衡量所提出的算法. 获得的激光测 量点模拟激光测距仪 Sick PLS 的参数模型, 其距离 分辨率为 $5 \mathrm{~cm}$, 角度分辨率为 $1^{\circ}$, 平均扫描距离为 $2.8 \mathrm{~m}$. 分别使用文献 [5] 中基于 Hough 密度谱的方 法 (HSM) 和本文提出的改进的基于 Hough 密度谱 的方法 (IHSM), 仅仅利用 Fourier-Mellin 变换来估 


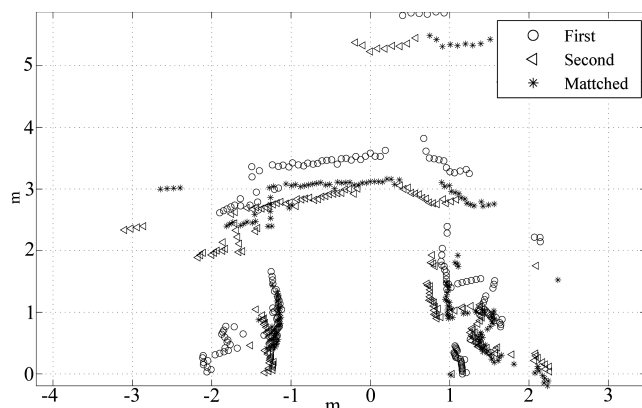

(a) HSM 方法

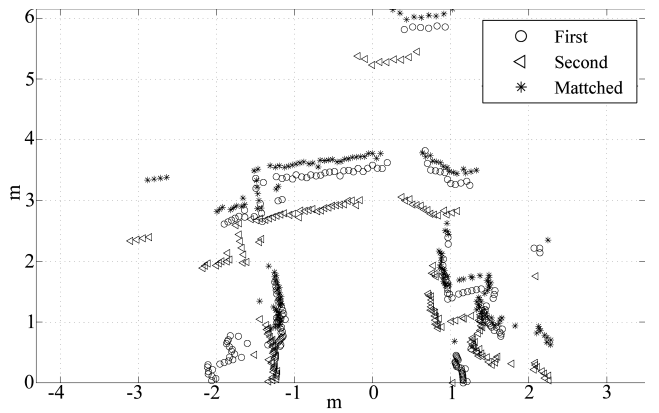

(c) FM 方法

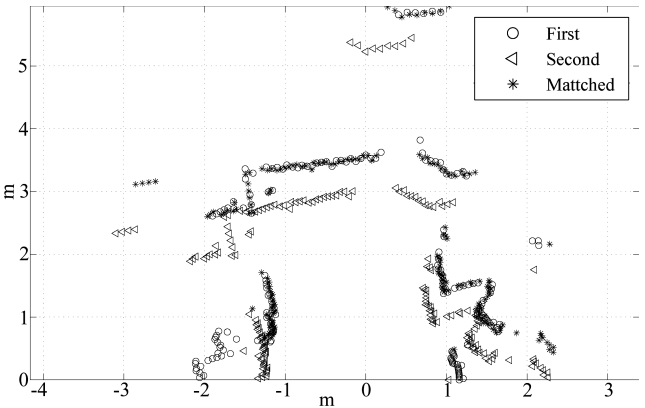

(b) IHSM 方法

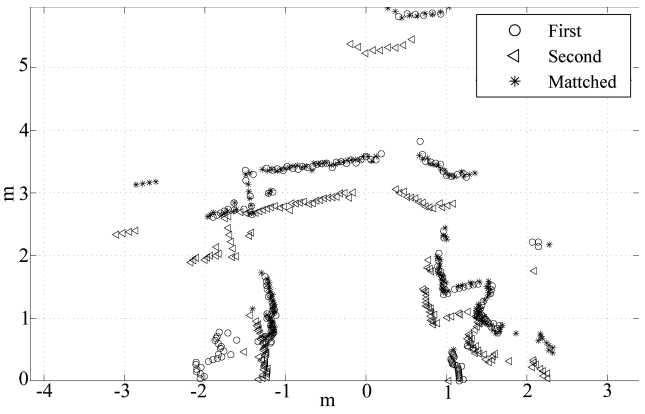

(d) FMH 方法

图 1 四种定位方法的匹配结果示意图

Fig. 1 The matching results of the four localization methods

计 $(R, T)$ 的方法 $(\mathrm{FM})$ 和改进的用 Fourier-Mellin 变换来估计 $R$ 、用基于 Hausdorff 距离的 ICP 算法 来估计 $T$ 的方法 $(\mathrm{FMH})$ 来估计机器人的当前位姿, 实验结果如表 1 所示. 由表 1 可以看出, 对于不 同的环境, 随着环境非结构化程度的增加, 误差会 有所增加. 对于相同的环境, IHSM 方法的角度误 差、平移误差以及匹配点在去掉外点后的 Hausdorff 距离均小于 HSM 方法; FM 方法的旋转角误差与 HSM 方法相比明显减小, 但对平移向量的估计改进 不大; $\mathrm{FMH}$ 方法的旋转角误差和平移误差较 HSM 方法均有较大改善; IHSM 方法与 FMH 方法的估 计精度相当. 这些试验结果表明: 与其它方法相 比, IHSM 方法和 FMH 方法能够更有效地提高移 动机器人的定位精度.

\section{2 实物实验}

实物实验以 iRobot 公司生产的 B21r 室内移 动机器人为研究对象. 它安装的是 Sick 公司的 PLS $101-312$ 型激光测距仪. 该激光测距仪 的距离分辨率为 $5 \mathrm{~cm}$, 角度分辨率为 $1^{\circ}$, 最远 射程可达 $50 \mathrm{~m}$. 控制机器人运动, 在运动前后可 获得两组激光测量点. 图 1 是分别使用 HSM 方 法、IHSM 方法、FM 方法以及 FMH 方法进行激 光测量点匹配的示意图. 在不考虑外点的情况 下，使用这四种方法匹配后的 Hausdorff 距离依次
为: $0.0716 \mathrm{~m} 、 0.0454 \mathrm{~m} 、 0.0675 \mathrm{~m} 、 0.0436 \mathrm{~m}$. 由图 1 可以看出, 与其它方法相比, IHSM 方法和 FMH 方法可更有效地应用在基于平面激光测量的移动机 器人定位中.

\section{6 结论}

把激光测量点的匹配问题这一时域问题转化 为频域问题来解决具有不需要初始估计、不存在特 征提取和对应问题、既可用于结构化环境又可用于 非结构化环境且对噪声不敏感等优点. 鉴于此, 本 文提出了两种基于平面激光测量的机器人自定位 的方法: 改进的基于 Hough 密度谱的方法和基于 Fourier-Mellin 变换的定位方法. 实验结果表明, 这 两种方法不仅保持了频域分析的优点, 而且精度要 高于 Andrea Censi 等在文献 [5] 中提出的方法, 是 两种较为有效的移动机器人定位方法, 具有一定的 实际应用价值.

\section{References}

1 Lingermann K, Surmann H, Nücher A, Hertzberg J. Indoor and outdoor localization for fast mobile robots. In: Proceedings of IEEE International Conference on Intelligrnt Robots and System. IEEE, Sendai, Japan, 2004. 2185 2190

2 Sooyong Lee, Iae-Bok Song. Mobile robot localization using 
range sensors: Consecutive scanning and cooperative scanning. International Journal of Control, Automation, and Systems, 2005, 3(1): 1 14

3 Feng Lu, Evangelos Milios. Robot pose estimation in unknown environments by matching $2 \mathrm{D}$ range scans. Journal of Intelligent and Robotic Systems, 1997, 18(5): 249 275

4 Minguez J, Lamiraux F, Montesano L. Metric-based scan matching algorithms for mobile robot displacement estimation. In: Proceedings of IEEE International Conference on Robotics and Automation. IEEE, Barcelona,Spain, 2005. 3557 3563

5 Andrea Censi, Luca Iocchi, Giorgio Grisetti. Scan matching in the Hough domain. In: Proceedings of IEEE International Conference on Robotics and Automation. IEEE, Barcelona, Spain, 2005, 2750 2755

6 Illingworth J, Kittler J. A survey of the Hough transform. Computer Vision, Graphics, and Image Processing , 1998, 40(1): $87 \sim 116$

7 Li Xiao-Ming, Zhao Xun-Po, Zheng Lian, Hu Zhan-Yi. An image registration technique based on Fourier-Mellin transform and its extended applications. Chinese Journal of Computers, 2006, 29(3): 466 472

(李晓明, 赵训坡, 郑链, 胡占义. 基于 Fourier-Mellin 变换的图像 配准及应用扩展. 计算机学报, 2006, 29(3): 466 472)

8 Zhao Xun-Po. A Study on Robust Image Curve Matching Techniques[Ph.D. dissertation], Beijing: Institute of $\mathrm{Au}-$ tomation, Chinese Academy of Sciences, 2004 (赵训坡. 鲁棒性图像曲线匹配方法研究 [博士学位论文], 北京: 中 国科学院自动化研究所, 2004)

9 Chen Qin-Sheng, Michel Defrise, Deconinck F. A symmetric phase-only matched filtering of Fourier-Mellin transforms for image registration and recognition. IEEE Transactions on Pattern Analysis and Machine Intelligence, 1994, 16(12): 1156 1168

10 Reddy B Srinivasa, Chatterji B N. An FFT-based technique for translation, rotation, and scale-invariant image registration. IEEE Transactions on Image Processing, 1996, 5(8): 1266 1271

11 Huttenlocher Daniel P, Klanderman Gregory A, Rucklidge William J. Comparing images using the Hausdorff distance. IEEE Transactions on Pattern Analysis and Machine Intelligence, 1993, 15(9): 850 863

12 Andrew Howard, Nicholas Roy. The Robotics Data Set Repository[Online], available: http://radish.sourceforge. net/, 2003

13 Richard Vaughan. Hosptial Floorplan Fort Sam Houston and Cave Bitmap[Online], available: http://cres.usc.edu/ radish/view-all.php, May 06, 2003

14 The Texas Speleological Survey, Cicuinacave[Online], available: http: //www. utexas. edu/tmm/sponsored_sites/tss/ CaveMaps/mapimages/cicurinacave.gif, May 22, 2005

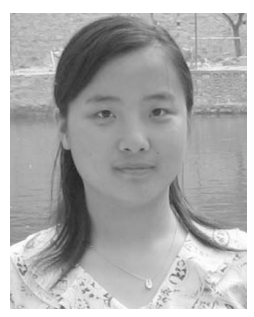

曾慧 2001 年和 2004 年分别获山东 大学控制学院学士学位和硕士学位, 现为中科院自动化研究所在读博士 生, 主要研究方向为机器人定位、视 觉测量等. 本文通信作者. E-mail: hzeng@nlpr.ia.ac.cn

(Zeng Hui Received her bachelor degree and master degree from Shandong University in 2001 and 2004 respectively. Currently, she is pursuing her $\mathrm{Ph}$. D. degree at Institute of Automation, Chinese Academy of Science. Her research interests include robot localization and visual metrology. Corresponding author of this paper.)

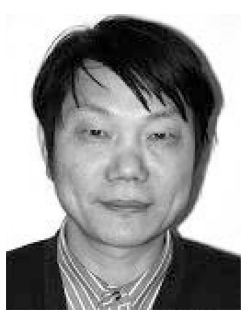

吴福朝 研究员，博士生导师，1995～ 1999 年为安徽大学人工智能研究所教 授, 2000 年起至今在中科院自动化研究 所工作，主要研究领域为摄像机标定、 三维重建和机器人自定位等. E-mail: fcwu@nlpr.ia.ac.cn

(Wu Fu-Chao Acted as a professor in Anhui University from 1995 to 1999. Since 2000, he has been a professor at Institute of Automation, Chinese Academy of Science. His research interests include camera calibration, 3D reconstruction, and robot self-localization.)

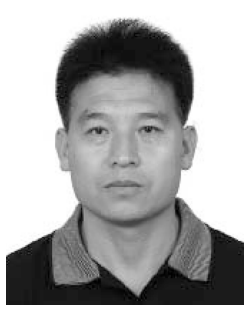

胡占义 研究员，博士生导师，1993 年 获比利时国家博士学位, 1993 年起至今 在中科院自动化研究所工作, 主要从事 摄像机标定、三维重建、Hough 变换和 视觉机器人导航等相关领域的研究工作. E-mail: huzy@nlpr.ia.ac.cn

(Hu Zhan-Yi Received his Ph.D. degree in computer science from University of Liege, Belgium in 1993. Since 1993, he has been a professor at Institute of Automation, Chinese Academy of Science. His research interests include camera calibration, 3D reconstruction, Hough transform, and vision guided robot navigation.) 